\title{
Charge doping and large lattice expansion in oxygen-deficient heteroepitaxial $\mathrm{WO}_{3}$
}

\author{
Giordano Mattoni, ${ }^{1, *}$ Alessio Filippetti, ${ }^{2,3}$ Nicola Manca, ${ }^{1}$ Pavlo Zubko, ${ }^{4,5}$ and Andrea D. Caviglia ${ }^{1}$ \\ ${ }^{1}$ Kavli Institute of Nanoscience, Delft University of Technology, 2628 CJ Delft, The Netherlands \\ ${ }^{2}$ IOM-CNR, S. P. Monserrato-Sestu km 0.7, Monserrato (CA) I-09042, Italy \\ ${ }^{3}$ Dipartimento di Fisica, Università di Cagliari, S. P. Monserrato-Sestu km 0.7, Monserrato (CA) I-09042, Italy \\ ${ }^{4}$ London Centre for Nanotechnology, University College London, 17-19 Gordon Street, London WC1H OHA, United Kingdom \\ ${ }^{5}$ Department of Physics and Astronomy, University College London, Gower Street, London WC1E 6BT, United Kingdom
}

(Received 14 November 2017; revised manuscript received 30 March 2018; published 15 May 2018)

\begin{abstract}
Tungsten trioxide $\left(\mathrm{WO}_{3}\right)$ is a versatile material with widespread applications ranging from electrochromics and optoelectronics to water splitting and catalysis of chemical reactions. For technological applications, thin films of $\mathrm{WO}_{3}$ are particularly appealing, taking advantage from a high surface-to-volume ratio and tunable physical properties. However, the growth of stoichiometric crystalline thin films is challenging because the deposition conditions are very sensitive to the formation of oxygen vacancies. In this paper, we show how background oxygen pressure during pulsed laser deposition can be used to tune the structural and electronic properties of $\mathrm{WO}_{3}$ thin films. By performing x-ray diffraction and low-temperature electrical transport measurements, we find changes in the $\mathrm{WO}_{3}$ lattice volume of up to $10 \%$ concomitantly with a resistivity drop of more than five orders of magnitude at room temperature as a function of increased oxygen deficiency. We use advanced $a b$ initio calculations to describe in detail the properties of the oxygen vacancy defect states and their evolution in terms of excess charge concentration. Our results depict an intriguing scenario where structural, electronic, optical, and transport properties of $\mathrm{WO}_{3}$ single-crystal thin films can all be purposely tuned by controlling the oxygen vacancy formation during growth.
\end{abstract}

DOI: 10.1103/PhysRevMaterials.2.053402

\section{INTRODUCTION}

Tungsten trioxide $\left(\mathrm{WO}_{3}\right)$ holds a special place in the family of complex oxides because its perovskite $\mathrm{ABO}_{3}$ lattice has an empty $A$ site. This characteristic determines an open crystalline structure, that is prone to host interstitial species which act as dopants for the otherwise insulating material [1-6]. For these reasons, $\mathrm{WO}_{3}$ finds wide use in electrochromic, optoelectronic, and gas-sensing applications [7-9]. Extensive research has been performed on $\mathrm{WO}_{3}$ films, amorphous layers, and nanorods [10-14]. The growth of single-crystal thin films has been recently demonstrated by means of several techniques, such as sputtering, molecular beam epitaxy, and pulsed laser deposition [15-19]. Since the structural and electronic properties of $\mathrm{WO}_{3}$ are very sensitive to oxygen stoichiometry [20-24], a precise control of oxygen partial pressure during the growth process is crucial to obtaining high quality thin films. Despite the several experimental and theoretical works investigating the characteristics of $\mathrm{WO}_{3}$ films under different growth conditions, a clear picture of the interplay among oxygen vacancy formation, crystalline quality, and electrical transport is missing so far.

In this paper, we study the effects of oxygen pressure during pulsed laser deposition (PLD) of $\mathrm{WO}_{3}$ thin films and show how it modifies the material's structural and electronic properties. X-ray diffraction (XRD) measurements reveal that heteroepitaxial $\mathrm{WO}_{3}$ thin films are in a tetragonal phase where the out-of-plane lattice parameter can be gradually

\footnotetext{
*g.mattoni@tudelft.nl
}

tuned up to $10 \%$, changing from $3.7 \AA$ measured in the most stoichiometric compound to $4.1 \AA$ in the most oxygendeficient case. By performing transport measurements, we find a semiconducting trend characterized by an activated transport regime with an energy gap that tends to zero with increasing oxygen vacancy concentration. We corroborate these findings with ab initio calculations, showing that oxygen vacancies are responsible for the formation of in-gap states, which donate electron carriers and increase the volume of the $\mathrm{WO}_{3}$ unit cell (uc). Our results constitute a comprehensive experimental and theoretical study of how oxygen defect states control the electronic and structural properties of $\mathrm{WO}_{3}$ thin films.

\section{FILM GROWTH}

To study the effect of background oxygen pressure $\left(p_{\mathrm{O}_{2}}\right)$ on $\mathrm{WO}_{3}$ thin-film growth by PLD, we prepare a series of samples deposited on top of $\mathrm{TiO}_{2}$-terminated $\mathrm{SrTiO}_{3}(001)$ substrates. We use a laser fluence of $1 \mathrm{~J} \mathrm{~cm}^{-2}$, a repetition rate of $1 \mathrm{~Hz}$, a target-to-sample distance of $55 \mathrm{~mm}$, and a fixed deposition duration of 1000 laser pulses. The growth temperature is $500{ }^{\circ} \mathrm{C}$, whereas $p_{\mathrm{O}_{2}}$ is varied in the range of 5-100 $\mu$ bar. The plasma plume is more diffused at lower $p_{\mathrm{O}_{2}}$ and more intense at higher $p_{\mathrm{O}_{2}}$ (see the Supplemental Material [25]). This is because the oxygen pressure in the PLD chamber influences the plume dynamics, enhancing scattering of the ablated species and thermalization with the background gas, so that the stoichiometry of the deposited material in general strongly depends upon $p_{\mathrm{O}_{2}}[26,27]$. Since $\mathrm{WO}_{3}$ is an oxide of a single cationic species, in this case $p_{\mathrm{O}_{2}}$ mainly affects the 

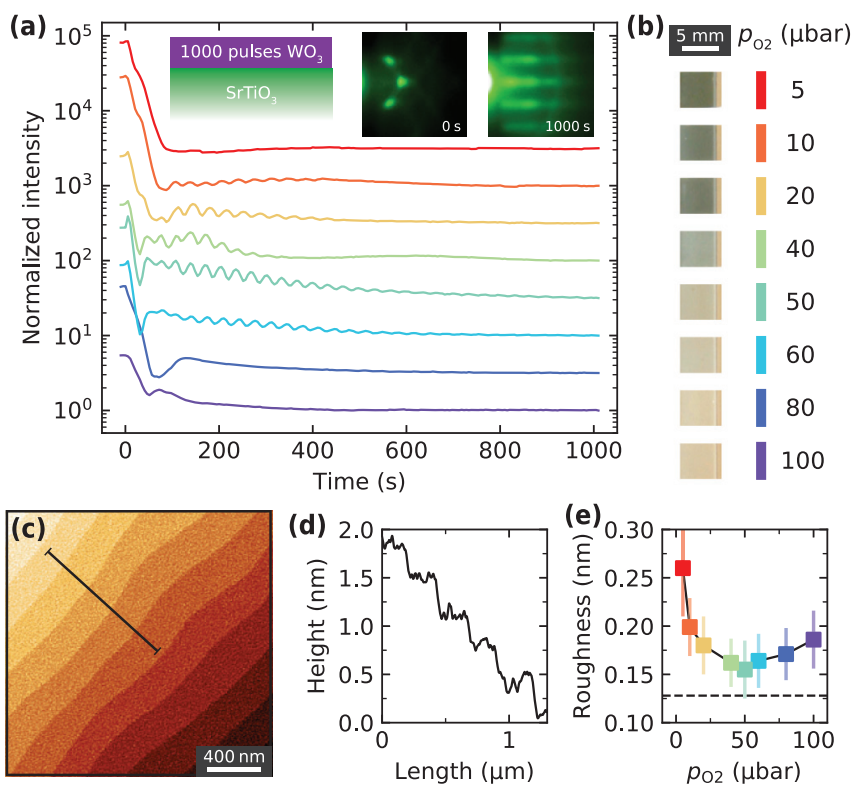

FIG. 1. Growth of $\mathrm{WO}_{3}$ thin films. (a) RHEED intensity oscillations during film growth at different values of $p_{\mathrm{O}_{2}}$ and (inset) heterostructure schematic, along with initial, and final RHEED diffraction patterns at $p_{\mathrm{O}_{2}}=50 \mu$ bar. (b) Photograph of the samples, (c) surface topography by atomic force microscopy, (d) line profile along the black line in (c), and (e) surface roughness calculated as the root mean square of the topographic signal.

oxidation state and the deposition rate of the growing film. We monitor the growth in situ by reflection high-energy electron diffraction (RHEED) and observe clear intensity oscillations when $p_{\mathrm{O}_{2}}$ is in the range of $10-60 \mu$ bar [Fig. 1(a)]. The RHEED pattern evolves from three well-defined diffraction points, typical of $\mathrm{SrTiO}_{3}$ single crystals [28], to a series of stripes that indicate bidimensional film growth. As shown in the photograph of Fig. 1(b), the sample color is also affected by the oxygen pressure, and it gradually changes from transparent to dark gray with decreasing $p_{\mathrm{O}_{2}}$. All the deposited $\mathrm{WO}_{3}$ films have a step-and-terrace surface morphology with single unit-cell steps [Figs. 1(c) and 1(d)]. This structure mimics the underlying $\mathrm{SrTiO}_{3}$ substrate, indicating uniform growth (further information in the Supplemental Material [25]). As shown in Fig. 1(e), the samples have very low surface roughness in the range of $0.15-0.25 \mathrm{~nm}$, which is comparable to the value of $0.13 \mathrm{~nm}$ that we measure on a pristine substrate. Interestingly, the roughness is minimal at $p_{\mathrm{O}_{2}}=50 \mu \mathrm{bar}$, pressure for which the RHEED oscillations during growth are more accentuated. These results indicate that for all $p_{\mathrm{O}_{2}}$ in the explored range the films grow with a smooth surface morphology.

\section{EXPERIMENTAL CHARACTERIZATION}

\section{A. X-ray diffraction}

To evaluate the crystal quality of the $\mathrm{WO}_{3}$ thin films, we perform $\mathrm{x}$-ray diffraction measurements. Figure 2(a) shows $\theta-2 \theta$ scans around the sharp (001) and (002) peaks of the $\mathrm{SrTiO}_{3}$ substrate. The $\mathrm{WO}_{3}$ film grown at $p_{\mathrm{O}_{2}}=100 \mu$ bar presents peaks at $2 \theta=24^{\circ}$ and $49^{\circ}$, surrounded by neat finitesize oscillations that demonstrate a high crystalline quality. No additional diffraction peak is observed, indicating that the thin films are in a single-crystal phase. For lower $p_{\mathrm{O}_{2}}$, the
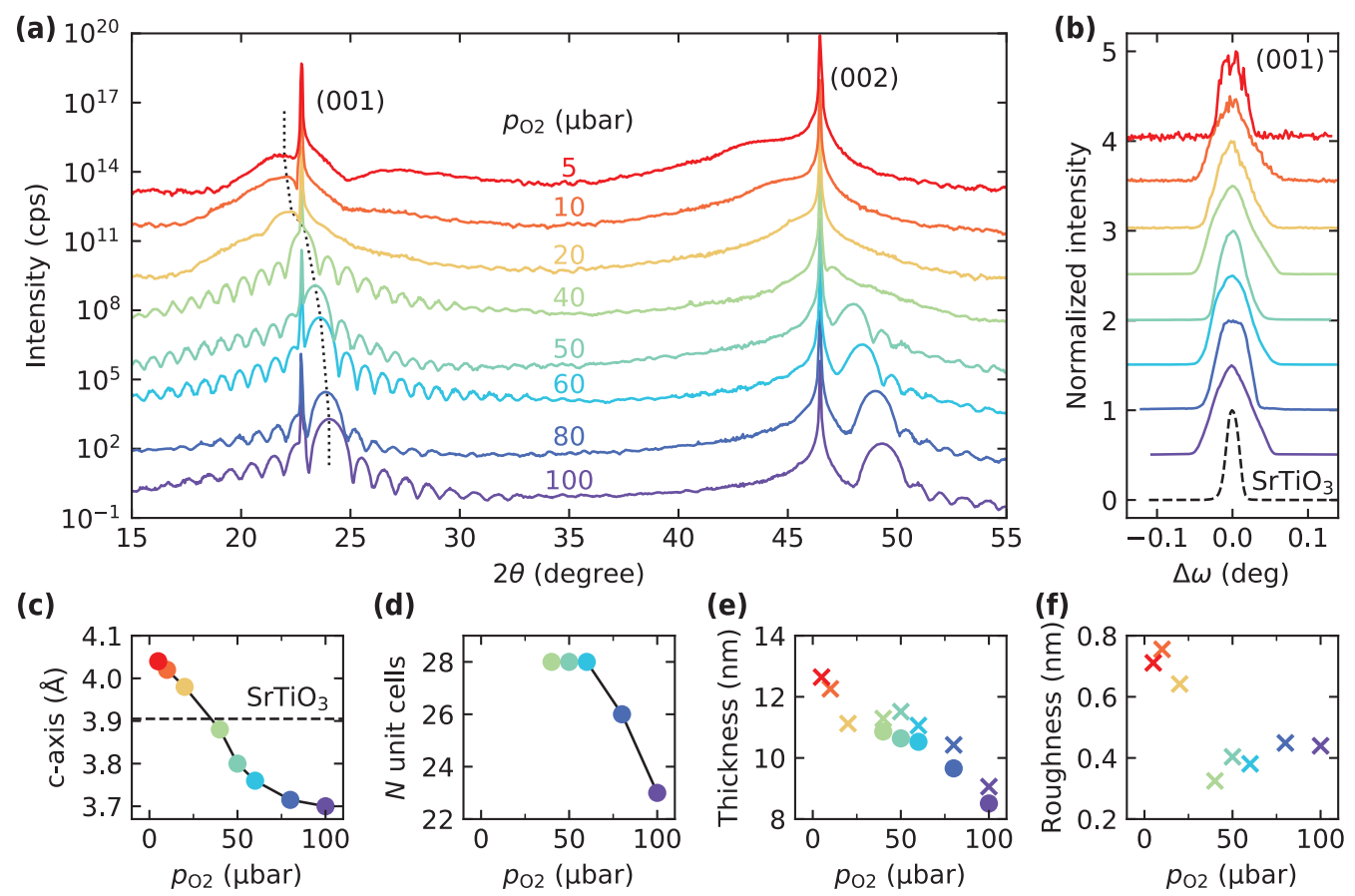

FIG. 2. X-ray diffraction characterization. (a) $\theta-2 \theta$ scans around the (001) and (002) peaks of the $\mathrm{SrTiO}_{3}$ substrate. The dotted line highlights the position of the $\mathrm{WO}_{3}(001)$ peak. (b) Comparison of the rocking curves around the $\mathrm{WO}_{3}(001)$ peak to the one around the substrate (001) peak (black dashed line). (c) Film $c$-axis parameter and substrate lattice constant (dashed line), (d) number of $\mathrm{WO}_{3}$ unit cells extracted by simulating the finite-size oscillations with a kinematic scattering model. (e) Film thickness and (f) roughness obtained from reflectivity measurements (crosses) and the $\theta-2 \theta$ data (circles). 
peaks and finite-size oscillations gradually shift to lower $2 \theta$ angles. At $p_{\mathrm{O}_{2}} \leqslant 20 \mu$ bar, the finite-size oscillations become less defined concurrent with a broadening of the diffraction peaks. Such signal degradation is a sign of decreased crystal quality, similar to what has been previously observed for highly doped $\mathrm{WO}_{3}[29,30]$. Considering the large lattice expansion observed at these values of $p_{\mathrm{O}_{2}}$, strain relaxation mediated by the formation of defects is likely to occur, contributing to the broadening of the XRD peaks.

In Fig. 2(b), we evaluate the presence of defects in $\mathrm{WO}_{3}$ by measuring rocking curves around the film (001) peak. We find for all samples a sharp peak with full width at half maximum between $0.02^{\circ}$ and $0.03^{\circ}$, very close to the value of $0.01^{\circ}$ found for the underlying $\mathrm{SrTiO}_{3}$ substrate. The sharp rocking curves indicate that the $\mathrm{WO}_{3}$ films have low mosaicity and present single-crystal quality. By performing reciprocal space maps (see the Supplemental Material [25]), we find that the in-plane lattice of all films is coherently strained to the substrate one $\left(a_{\mathrm{WO}_{3}}=a_{\mathrm{SrTiO}_{3}}=3.905 \AA\right)$. By using Bragg's law, we extract in Fig. 2(c) the out-of-plane $c$-axis parameter from the $2 \theta$ position of $\mathrm{WO}_{3}$ diffraction peaks. At $p_{\mathrm{O}_{2}}=$ $100 \mu$ bar we obtain $c=3.70 \AA$, whereas samples grown at lower $p_{\mathrm{O}_{2}}$ present a larger $c$-axis parameter [Fig. 2(c)]. Because all films are epitaxially locked in plane to the substrate lattice, this indicates an increase in $\mathrm{WO}_{3}$ unit-cell volume, a trend compatible with a higher concentration of oxygen vacancies as previously reported for oxygen-deficient thin films grown by PLD [31-35]. For $p_{\mathrm{O}_{2}}<40 \mu$ bar, the $c$ axis becomes bigger than $a_{\mathrm{SrTiO}_{3}}$, signaling a transition from tensile to compressive strain. We note that, concomitant with this crossover, the finite-size oscillations disappear, suggesting that films grown at $p_{\mathrm{O}_{2}}<40 \mu$ bar have a lower crystal quality.

By simulating the finite-size fringes of the XRD data with a kinematic scattering model, we extract in Fig. 2(d) the number of unit cells $N_{\mathrm{WO}_{3}}$ forming the thin films (further details in the Supplemental Material [25]). We find a constant $N_{\mathrm{WO}_{3}}=28$ uc for $p_{\mathrm{O}_{2}} \leqslant 60 \mu \mathrm{bar}$, and lower values at higher oxygen pressures. Considering that all films were deposited with the same total number of laser pulses, we associate the decreased $\mathrm{N}_{\mathrm{WO}_{3}}$ with enhanced scattering of the plasma plume at higher pressures, which reduces the amount of material deposited on the substrate. For $p_{\mathrm{O}_{2}} \leqslant 20 \mu$ bar, the absence of finite-size oscillations does not allow to determine the number of unit cells by $\theta-2 \theta$ measurements. We thus perform $\mathrm{x}$-ray reflectivity measurements from which we extract the film thickness and roughness indicated by the crosses in Figs. 2(e) and 2(f) (raw data in the Supplemental Material [25]). The total film thickness shows an increasing trend with lower pressure, which is a combined effect of the $c$-axis expansion and decreased plume scattering. In Fig. 2(e) we also evaluate the film thickness from the $\theta-2 \theta$ measurements as $c \times N_{\mathrm{WO}_{3}}$ (circles), finding good agreement with the reflectivity data. Concerning the film roughness in Fig. 2(f), we observe a minimum at $p_{\mathrm{O}_{2}}=40 \mu \mathrm{bar}$, consistent with what obtained from the topography data of Fig. 1(e). These measurements show that the $\mathrm{WO}_{3} c$-axis parameter can be changed up to $10 \%$ by tuning the oxygen pressure during growth, whereas preserving the in-plane match with the substrate lattice and a flat surface.
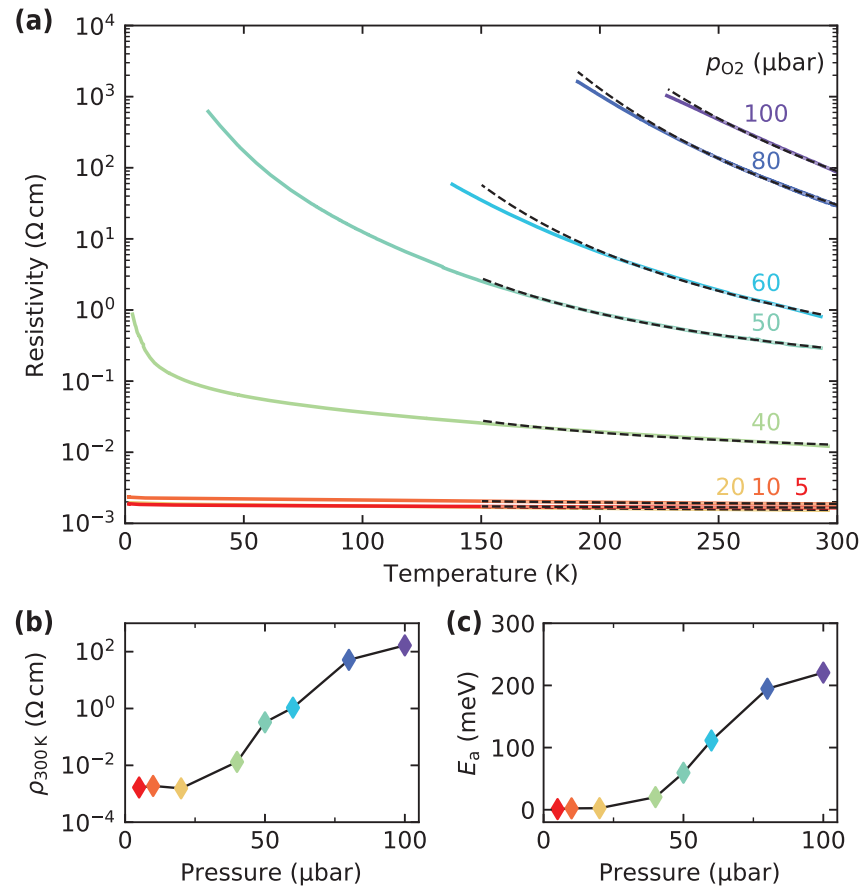

FIG. 3. Resistivity measurements. (a) Four-probe resistivity vs temperature curves. The dashed lines are best fits to the Arrhenius model for activated transport in Eq. (1). (b) Room-temperature resistivity and (c) activation energy for transport extracted from the fits.

\section{B. Electrical transport}

We now investigate the effect of oxygen vacancies on $\mathrm{WO}_{3}$ electronic properties by measuring the resistivity vs temperature characteristic of samples grown in different values of $p_{\mathrm{O}_{2}}$. The measurements are performed in a van der Pauw configuration, and electrical contact to $\mathrm{WO}_{3}$ is obtained with ultrasonically bonded $\mathrm{Al}$ wires. All the samples show a semiconducting behavior [Fig. 3(a)] with the resistivity monotonously increasing upon lowering temperature. The resistivity $\rho$ is calculated using the film thickness measured by $\mathrm{x}$-ray reflectivity. Lower $p_{\mathrm{O}_{2}}$ results in flatter curves with lower room-temperature resistivity $\left(\rho_{300 \mathrm{~K}}\right)$ as reported in Fig. 3(b). The data show a sigmoid trend with a variation of more than five orders of magnitude in the studied range. Such a trend is comparable to the one observed for the $c$-axis parameter in Fig. 2(c), suggesting the existence of a direct correlation between the lattice expansion and the electronic doping. We describe the transport data using an Arrhenius-type behavior,

$$
\sigma(T)=\frac{1}{\rho(T)} \propto \exp \left(-\frac{E_{\mathrm{a}}}{k_{\mathrm{B}} T}\right),
$$

where $E_{\mathrm{a}}$ is the activation energy for charge transport. The experimental curves are fit from room temperature down to $150 \mathrm{~K}$ [dashed lines in Fig. 3(a)], showing good agreement (fits over a broader temperature range are discussed in the Supplemental Material [25]). We extract $E_{\mathrm{a}} \sim 220 \mathrm{meV}$ at the highest $p_{\mathrm{O}_{2}}$, which is about one order of magnitude smaller than the optical band of $3 \mathrm{eV}$ found in bulk $\mathrm{WO}_{3}[20,36]$. This suggests that the observed activated transport arises from localized states lying inside the $\mathrm{WO}_{3}$ band gap, which form to 
some extent also at the highest $p_{\mathrm{O}_{2}}$ value. At lower $p_{\mathrm{O}_{2}}$, the concentration of oxygen vacancies in the film increases and determines a vanishing $E_{\mathrm{a}}$. Even though the $\mathrm{WO}_{3}$ films are on the verge of an insulator-to-metal transition, we measure semiconducting behavior also at the highest vacancy concentration. This is different from what was observed for other doping mechanisms where a metallic state was achieved at high doping levels [4,20,24,37]. The comparison with ab initio calculations, reported in the next section, indicates that reduced crystal quality and increased disorder during low-pressure PLD growth are the most probable causes for the persistent semiconducting state measured in our most doped $\mathrm{WO}_{3}$ thin films.

To probe the density of the charge carriers, we perform low-temperature magnetotransport measurements. Since most of the samples are highly resistive, reliable Hall measurements can be obtained only for $p_{\mathrm{O}_{2}} \leqslant 20 \mu$ bar. In this high-doping condition, all the samples show similar magnetotransport data (see Supplemental Material [25]) from which we extract a carrier density of $n_{3 \mathrm{D}}=4 \times 10^{21} \mathrm{~cm}^{-3}$ and a mobility of $\mu=0.6 \mathrm{~cm} \mathrm{~V}^{-1} \mathrm{~s}^{-1}$ at $1.5 \mathrm{~K}$, in agreement with previous reports of oxygen-deficient $\mathrm{WO}_{3}$ materials [23,24]. The measured electron density corresponds to about 0.25 electrons per unit cell, which would be equivalent to an oxygen vacancy concentration of about $4 \%$ if these are the only source of electron donors.

\section{AB INITIO CALCULATIONS}

To investigate the origin of the observed structural and transport behavior of $\mathrm{WO}_{3}$ films, we perform $a b$ initio calculations by variational pseudo-self-interaction-corrected (VPSIC) density functional theory (DFT) [38-41]. To validate our results, we first calculated several $\mathrm{WO}_{3}$ bulk phases (further details in the Supplemental Material [25]), finding good agreement with the existing literature $[6,42]$. The growth on $\mathrm{SrTiO}_{3}$ substrates was simulated using pseudocubic supercells with an in-plane lattice constant fixed at $a_{\mathrm{SrTiO}_{3}}=3.905 \AA$ and the orthogonal axis left free to relax. The calculations show that the stress associated with the planar expansion is partially relieved with a shrinkage of the out-of-plane lattice parameter to $c=3.685 \AA$ (Table I). This value is comparable with the experimental data of $\mathrm{WO}_{3}$ films grown at the highest oxygen pressure reported in Fig. 2(c), which are thus identified as stoichiometric.

Starting from the strained $\mathrm{WO}_{3}$ structure, we introduce vacancies by removing oxygen atoms on the $\mathrm{WO}_{2}$ crystal planes. We study oxygen vacancy concentrations of $2 \%\left(\mathrm{WO}_{2.94}\right)$ and $4 \%\left(\mathrm{WO}_{2.88}\right)$. After a further structural relaxation, our

TABLE I. A $b$ initio results. Properties from bulk $\delta-\mathrm{WO}_{3}$ and for films strained in plane to the $\mathrm{SrTiO}_{3}$ substrate lattice with different concentrations of oxygen vacancies on the $\mathrm{WO}_{2}$ planes. The effective mass $m^{*}$ is calculated along the planar $\Gamma-M$ direction.

\begin{tabular}{lcccc}
\hline \hline & $\mathrm{Bulk} \delta-\mathrm{WO}_{3}$ & $\mathrm{WO}_{3}$ & $\mathrm{WO}_{2.94}$ & $\mathrm{WO}_{2.88}$ \\
\hline O vacancy & $0 \%$ & $0 \%$ & $2 \%$ & $4 \%$ \\
$c(\AA)$ & 3.75 & 3.685 & 3.72 & 3.73 \\
$E_{\mathrm{g}}(\mathrm{eV})$ & 2.71 & 2.83 & & \\
$m^{*}\left(m_{\mathrm{e}}\right)$ & 0.56 & 0.85 & 2.41 & 1.16 \\
\hline \hline
\end{tabular}

calculations find lattice increases to $c=3.72$ and $c=3.73 \AA$ for the two vacancy concentrations (Table I). This trend is in qualitative agreement with our experimental results of Fig. 2. However, the calculated values are considerably lower than what we measure for films grown at low pressures, a discrepancy that can be explained considering another result from our calculations. Previous literature reports show that the lattice expansion due to oxygen vacancies is maximum when the charge is fully localized $[43,44]$. As discussed in the following paragraph, our DFT calculations predict that $\mathrm{WO}_{3}$ grown at the lowest $p_{\mathrm{O}_{2}}$ should be metallic and thus have a limited lattice expansion. However, this is not observed in our transport experiments where we measure a semiconducting behavior and, consistently, higher lattice expansion. Furthermore, temperaturerelated effects, which are not included in our calculations, could be responsible for the higher lattice volume [45].

In Fig. 4(a) we show the calculated in-plane band structure for strained $\mathrm{WO}_{3}$ films with different oxygen vacancy concentrations (extended plots in the Supplemental Material [25]). A single oxygen vacancy generates an excess of two electrons. In the limit of an isolated point defect, this charge remains trapped by the $\mathrm{W}$ atoms surrounding the vacancy, which change their ionic charge from the stoichiometric $\mathrm{W}^{6+}$ to a lower value, also giving rise to color centers responsible for the photochromic effect $[7,46]$. Localization may eventually be strengthened by the formation of small polarons [46]. According to our calculations, the excess charge is reversed into the $t_{2 \mathrm{~g}}$ orbitals of the $\mathrm{W}$ atoms, forming a shallow band right below the bottom of the conduction band [orange dash-dotted curves in Fig. 4(a)]. Although this band is optically separated from the bulklike $t_{2 \mathrm{~g}}$ conduction bands running above it, the system is metallic for both examined doping levels as indicated by the position of the Fermi energy [green dashed lines in Fig. 4(a)]. The defect state can be visualized in real space through the isosurface plot of Fig. 4(b): The defect charge is mostly localized on the two $\mathrm{W}$ atoms nearest neighbors of the vacancy with clear $t_{2 \mathrm{~g}}$ symmetry. This is also seen by the broader bandwidth of the vacancy state along the diagonal direction $X-M$, characteristic of $t_{2 \mathrm{~g}}$ orbitals, instead of the cubic edge direction $\Gamma-X$.

In Table I we report the band gaps and effective masses along the planar $\Gamma-M$ direction. The vacancy-free structure shows an insulating state with a direct gap $E_{\mathrm{g}}=2.83 \mathrm{eV}$ at the $\Gamma$ point, substantially larger than the indirect gap in bulk $\delta-\mathrm{WO}_{3}$ of $2.71 \mathrm{eV}$. This is a consequence of the tensile strain present in the structure which stretches the planar bonds and reduces the $t_{2 \mathrm{~g}}$ bandwidth as evidenced by the increased effective mass. Even though the $\mathrm{WO}_{2.94}$ and $\mathrm{WO}_{2.88}$ structures have small optical gaps at $\Gamma$ of 75 and $150 \mathrm{meV}$, respectively, they show metallic character in transport due to the broader density of states in the orthogonal direction [25]. With more excess charge, the gaps and effective masses are progressively reduced. This is due to electronic correlation and band filling effects, which cause a spread in the bandwidth with respect to the empty conduction bands of the undoped system. For lower vacancy concentration, up to the limit of the isolated point defect, instead, the carrier density is expected to be reduced, leading to the formation of an insulating state.

In order to theoretically estimate electron mobility and resistivity in a wider range of vacancy concentrations, we apply the Bloch-Boltzmann approach to mix the ab initio band 



(b)


FIG. 4. DFT calculations of heteroepitaxial $\mathrm{WO}_{3}$ films with oxygen vacancies. (a) Left panels: in-plane band structure for $\mathrm{WO}_{3}$ with $0 \%, 2 \%$, and $4 \%$ of oxygen vacancies. The calculations are performed using $2 \times 2 \times 4$ supercells with an oxygen vacancy on the $\mathrm{WO}_{2}$ planes. The band of the oxygen defect states is indicated by the orange dash-dotted line, and the Fermi level is represented by the green dashed line. Right panels: density of states projected on the oxygen $2 p$ (red) and tungsten $5 d$ (gray) orbitals. For $\mathrm{WO}_{2.94}$ the horizontal solid lines show the Fermi level corresponding to carrier densities of $n_{1.5 \mathrm{~K}}=5 \times 10^{21} \mathrm{~cm}^{-3}$ (green) and $1 \times 10^{18} \mathrm{~cm}^{-3}$ (violet). (b) Computed $2 \times 1 \times 1$ supercell of WO 2.94 showing the real-space charge-density isosurface of the defect band (orange). The oxygen vacant site at the bottom is indicated by the dashed red circle. (c) Mobility as a function of carrier density (circles) at $1.5 \mathrm{~K}$ from the DFT calculations. The density at the Fermi level is indicated by the white circle. The diamond represents the experimental data from Hall effect measurements for the sample grown at $p_{\mathrm{O}_{2}}=20 \mu$ bar. (d) Resistivity vs temperature curves for $\mathrm{WO}_{2.94}$ from the DFT calculations (the green dashed line corresponds to the Fermi level).

energies of the $\mathrm{WO}_{2.94}$ structure with a model description of the electron-phonon scattering $[47,48]$. We tune the Fermi level in a rigid band approximation to simulate the variation in the excess carrier density produced by the vacancies. Results of three-dimensional averages of mobility vs carrier density at $1.5 \mathrm{~K}$ are reported in Fig. 4(c). As a consequence of the increasing population of high-energy $t_{2 \mathrm{~g}}$ bands of $\mathrm{WO}_{3}$, the mobility increases with the carrier density and, for $2 \%$ vacancies, we find $n=2.2 \times 10^{21} \mathrm{~cm}^{-3}$ and $\mu=30 \mathrm{~cm}^{2} \mathrm{~V}^{-1} \mathrm{~s}^{-1}$ [white circle in Fig. 4(c)]. In our oxygen-deficient films, we measure a Hall mobility of $\mu=0.5 \mathrm{~cm}^{2} \mathrm{~V}^{-1} \mathrm{~s}^{-1}$ (orange diamond in Fig. 4(c) and raw data in the Supplemental Material [25]) which, in our calculations, would be achieved for a considerably lower carrier density $n=1 \times 10^{18} \mathrm{~cm}^{-3}$. By using the $a b$ initio transport coefficients, we calculate the corresponding resistivity vs temperature in Fig. 4(d). At densities below $n=1 \times 10^{19} \mathrm{~cm}^{-3}$, we observe an insulating transport regime for which only the energy-flat lowest portion of the defect state is populated with vanishing band velocities. In this regime, the electrical transport is thermally activated, similar to what we measure experimentally. Above $n=1 \times 10^{19} \mathrm{~cm}^{-3}$, a metallic state is observed in our calculations, but it is not achieved experimentally. This is a clear indication that disorder in our thin films plays a crucial role in preventing the occurrence of an insulator-to-metal transition, consistent with the experimental observation of a low electron mobility.
Several aspects of our transport calculations are in good agreement with our measurements. In particular, the amplitude of resistivity vs temperature matches well the experimental data, and the shallow temperature dependence of the resistivity is experimentally verified for most of the doped samples. On the other hand, there is an offset between the calculated and the Hall mobility corresponding to the measured carrier density, the latter being two orders of magnitude lower. This discrepancy indicates that lattice disorder in the samples grown at low $p_{\mathrm{O}_{2}}$ reduces the mobility and determines the observed semiconducting character.

\section{CONCLUSIONS}

To summarize, we reported how the structural, electronic, and transport properties of $\mathrm{WO}_{3}$ thin films are affected by changes in the oxygen pressure during PLD growth. The outof-plane lattice constant of our thin films increases up to $10 \%$ as a consequence of the introduction of oxygen defects, whereas a pseudocubic phase with single-crystal quality is maintained. Oxygen vacancies act as electron donors and dope the material. Our VPSIC-DFT calculations describe oxygen vacancies in strained $\mathrm{WO}_{3}$ films as weakly localized shallow donors of $t_{2 \mathrm{~g}}$ orbital character, lying only a few tens of meV below the bulklike $t_{2 \mathrm{~g}}$ mobility edge. According to our Bloch-Boltzmann rigid-band calculations, full localization is only reached for 
$n<1 \times 10^{19} \mathrm{~cm}^{-3}$, corresponding to an extremely small vacancy concentration of $0.01 \%$. The measurements are in good qualitative agreement with our ab initio calculations, showing that a higher concentration of oxygen vacancies results in an expansion of the $\mathrm{WO}_{3}$ lattice and reduces the energy gap regulating the electronic transport. Additional effects, such as disorder and defect clustering, are likely to enhance the charge localization and could be responsible for a higher threshold of vacancy concentration separating charge-localized and chargedelocalized regimes. Our results constitute a fundamental step towards understanding and engineering the properties of a material which is likely destined to become a rising star in the energy and nanoelectronic applications of the future.

\section{ACKNOWLEDGMENTS}

This work was supported by The Netherlands Organisation for Scientific Research (NWO/OCW) as part of the Frontiers of Nanoscience Program (NanoFront) and by the Dutch Foundation for Fundamental Research on Matter (FOM). The research leading to these results has received funding from the European Research Council under the European Union's H2020 Programme/ ERC Grant Agreement No. [677458].
[1] C. G. Granqvist, Sol. Energy Mater. Sol. Cells 60, 201 (2000).

[2] R. Chatten, A. V. Chadwick, A. Rougier, and P. J. Lindan, J. Phys. Chem. B 109, 3146 (2005).

[3] D. Hirai, E. Climent-Pascual, and R. J. Cava, Phys. Rev. B 84, 174519 (2011).

[4] N. Haldolaarachchige, Q. Gibson, J. Krizan, and R. J. Cava, Phys. Rev. B 89, 104520 (2014).

[5] T. Soma, K. Yoshimatsu, and A. Ohtomo, Appl. Phys. Express 9, 075801 (2016).

[6] H. Hamdi, E. K. H. Salje, P. Ghosez, and E. Bousquet, Phys. Rev. B 94, 245124 (2016).

[7] S. K. Deb, Sol. Energy Mater. Sol. Cells 92, 245 (2008).

[8] H. Long, W. Zeng, and H. Zhang, J. Mater. Sci.: Mater. Electron. 26, 4698 (2015).

[9] S. Cong, F. Geng, and Z. Zhao, Adv. Mater. 28, 10518 (2016).

[10] L. J. LeGore, O. D. Greenwood, J. W. Paulus, D. J. Frankel, and R. J. Lad, J. Vac. Sci. Technol. A 15, 1223 (1997).

[11] P. Tägtström and U. Jansson, Thin Solid Films 352, 107 (1999).

[12] A. Garg, J. Leake, and Z. Barber, J. Phys. D 33, 1048 (2000).

[13] O. Hussain, A. Swapnasmitha, J. John, and R. Pinto, Appl. Phys. A 81, 1291 (2005).

[14] M. Mamun, K. Zhang, H. Baumgart, and A. Elmustafa, ECS J. Solid State Sci. Technol. 4, P398 (2015).

[15] A. Braun, F. Aksoy Akgul, Q. Chen, S. Erat, T.-W. Huang, N. Jabeen, Z. Liu, B. S. Mun, S. S. Mao, and X. Zhang, Chem. Mater. 24, 3473 (2012).

[16] Y. Du, M. Gu, T. Varga, C. Wang, M. E. Bowden, and S. A. Chambers, ACS Appl. Mater. Interfaces 6, 14253 (2014).

[17] X. Leng, J. Pereiro, J. Strle, A. T. Bollinger, and I. Božović, APL Mater. 3, 096102 (2015).

[18] H. Kalhori, S. B. Porter, A. S. Esmaeily, M. Coey, M. Ranjbar, and H. Salamati, Appl. Surf. Sci. 390, 43 (2016).

[19] A. Herklotz, S. F. Rus, S. Kc, V. R. Cooper, A. Huon, E.-J. Guo, and T. Z. Ward, APL Mater. 5, 066106 (2017).

[20] W. Sahle and M. Nygren, J. Solid State Chem. 48, 154 (1983).

[21] S. C. Moulzolf, S.-a. Ding, and R. J. Lad, Sens. Actuators, B 77, 375 (2001).

[22] X. He, Y. Yin, J. Guo, H. Yuan, Y. Peng, Y. Zhou, D. Zhao, K. Hai, W. Zhou, and D. Tang, Nanoscale Res. Lett. 8, 50 (2013).

[23] X. Meng, F. Quenneville, F. Venne, E. Di Mauro, D. Ișık, M. Barbosa, Y. Drolet, M. M. Natile, D. Rochefort, F. Soavi et al., J. Phys. Chem. C 119, 21732 (2015).

[24] S. G. Altendorf, J. Jeong, D. Passarello, N. B. Aetukuri, M. G. Samant, and S. S. Parkin, Adv. Mater. 28, 5284 (2016).
[25] See Supplemental Material at http://link.aps.org/supplemental/ 10.1103/PhysRevMaterials.2.053402 for additional information on the PLD plasma plume, film characterization, (magneto)transport measurements at different temperatures, and $a b$ initio calculations, which includes Refs. [42,49-57].

[26] S. Wicklein, A. Sambri, S. Amoruso, X. Wang, R. Bruzzese, A. Koehl, and R. Dittmann, Appl. Phys. Lett. 101, 131601 (2012).

[27] D. J. Groenendijk, N. Manca, G. Mattoni, L. Kootstra, S. Gariglio, Y. Huang, E. van Heumen, and A. D. Caviglia, Appl. Phys. Lett. 109, 041906 (2016).

[28] J. H. Haeni, C. D. Theis, and D. G. Schlom, J. Electroceram. 4, 385 (2000).

[29] Y. He, M. Gu, H. Xiao, L. Luo, Y. Shao, F. Gao, Y. Du, S. X. Mao, and C. Wang, Angew. Chem., Int. Ed. 55, 6244 (2016).

[30] Z. Wang, Y. He, M. Gu, Y. Du, S. X. Mao, and C. Wang, ACS Appl. Mater. Interfaces 8, 24567 (2016).

[31] S. McIntosh, J. F. Vente, W. G. Haije, D. H. Blank, and H. J. Bouwmeester, Chem. Mater. 18, 2187 (2006).

[32] E. Choi, J. Kim, D. Cuong, and J. Lee, in 17th IEEE International Symposium on the Applications of Ferroelectrics, Santa Re, NM, USA, 2008 (IEEE, 2008), Vol. 1, pp. 1-2.

[33] A. J. Hauser, E. Mikheev, N. E. Moreno, J. Hwang, J. Y. Zhang, and S. Stemmer, Appl. Phys. Lett. 106, 092104 (2015).

[34] M. Lorenz, G. Wagner, V. Lazenka, P. Schwinkendorf, M. Bonholzer, M. J. Van Bael, A. Vantomme, K. Temst, O. Oeckler, and M. Grundmann, Materials 9, 44 (2016).

[35] E. Enriquez, A. Chen, Z. Harrell, P. Dowden, N. Koskelo, J. Roback, M. Janoschek, C. Chen, and Q. Jia, Sci. Rep. 7, 46184 (2017).

[36] D. W. Bullett, J. Phys. C 16, 2197 (1983).

[37] K. Yoshimatsu, T. Soma, and A. Ohtomo, Appl. Phys. Express 9, 075802 (2016).

[38] A. Filippetti, C. D. Pemmaraju, S. Sanvito, P. Delugas, D. Puggioni, and V. Fiorentini, Phys. Rev. B 84, 195127 (2011).

[39] T. Archer, C. D. Pemmaraju, S. Sanvito, C. Franchini, J. He, A. Filippetti, P. Delugas, D. Puggioni, V. Fiorentini, R. Tiwari, and P. Majumdar, Phys. Rev. B 84, 115114 (2011).

[40] D. Puggioni, A. Filippetti, and V. Fiorentini, Phys. Rev. B 86, 195132 (2012).

[41] P. Delugas, A. Filippetti, A. Gadaleta, I. Pallecchi, D. Marré, and V. Fiorentini, Phys. Rev. B 88, 115304 (2013).

[42] F. Wang, C. Di Valentin, and G. Pacchioni, J. Phys. Chem. C 115, 8345 (2011). 
[43] D. Marrocchelli, S. Bishop, H. Tuller, G. Watson, and B. Yildiz, Phys. Chem. Chem. Phys. 14, 12070 (2012).

[44] D. Marrocchelli, S. Bishop, and J. Kilner, J. Mater. Chem. A 1, 7673 (2013).

[45] A. Glensk, B. Grabowski, T. Hickel, and J. Neugebauer, Phys. Rev. X 4, 011018 (2014).

[46] K. Bange, Sol. Energy Mater. Sol. Cells 58, 1 (1999).

[47] A. Filippetti, P. Delugas, M. J. Verstraete, I. Pallecchi, A. Gadaleta, D. Marré, D. F. Li, S. Gariglio, and V. Fiorentini, Phys. Rev. B 86, 195301 (2012).

[48] P. Delugas, A. Filippetti, M. J. Verstraete, I. Pallecchi, D. Marré, and V. Fiorentini, Phys. Rev. B 88, 045310 (2013).

[49] G. Catalan, R. M. Bowman, and J. M. Gregg, Phys. Rev. B 62, 7892 (2000).

[50] P. González-Borrero, F. Sato, A. Medina, M. L. Baesso, A. C. Bento, G. Baldissera, C. Persson, G. A. Niklasson, C. G.
Granqvist, and A. Ferreira da Silva, Appl. Phys. Lett. 96, 061909 (2010).

[51] M. B. Johansson, G. Baldissera, I. Valyukh, C. Persson, H. Arwin, G. A. Niklasson, and L. Österlund, J. Phys.: Condens. Matter 25, 205502 (2013).

[52] P. Woodward, A. Sleight, and T. Vogt, J. Phys. Chem. Solids 56, 1305 (1995).

[53] A. van de Walle and G. Ceder, Phys. Rev. B 59, 14992 (1999).

[54] P. Haas, F. Tran, and P. Blaha, Phys. Rev. B 79, 085104 (2009).

[55] D. G. Barton, M. Shtein, R. D. Wilson, S. L. Soled, and E. Iglesia, J. Phys. Chem. B 103, 630 (1999).

[56] H. Zheng, J. Z. Ou, M. S. Strano, R. B. Kaner, A. Mitchell, and K. Kalantar-zadeh, Adv. Funct. Mater. 21, 2175 (2011).

[57] R. Vemuri, M. H. Engelhard, and C. Ramana, ACS Appl. Mater. Interfaces 4, 1371 (2012). 